\title{
An Incidentally Found True Portal Vein Aneurysm Secondary to Left Liver Lobe Aplasia in a Non-Cirrhotic Pregnant Woman Presented With Ovarian Torsion
}

\author{
Ahmed Said Cil $^{\mathrm{a}}$, Murat Bozkurt ${ }^{\mathrm{b}, \mathrm{d}}$, Duygu Kara Bozkurt ${ }^{\mathrm{c}}$
}

\begin{abstract}
We report a rare case of coexistence of true portal vein aneurysm (PVA) with left liver lobe aplasia in a non-cirrhotic pregnant woman presented with ovarian torsion. We discussed the clinical and radiologic findings of a true PVA with left liver lobe aplasia found incidentally in a 27 -week pregnant woman presented with acute abdomen and operated for a right side ovarian torsion. Portal vein diameter measured over $20 \mathrm{~mm}$ is called as PVA. PVA is an uncommon entity, and most of these aneurysms are acquired and detected in patients with underlying hepatocellular disease and portal hypertension. To the best of our knowledge, there is no report about PVA development secondary to left liver lobe aplasia in English literature. We found this association incidentally in a pregnant woman with normal hepatic parenchyma and no findings of portal hypertension.
\end{abstract}

Keywords: Portal vein aneurysm; Pregnancy; Ovarian torsion, Color Doppler ultrasound; Magnetic resonance imaging

\section{Introduction}

There are many reports on hepatic lobe agenesis, hyperplasia and portal vein anomalies in English literature [1]. However, to the best of our knowledge, there is no report on an aneurismal dilation of portal vein due to left liver lobe aplasia. Portal vein aneurysms (PVAs) are commonly observed as sac-

\footnotetext{
Manuscript accepted for publication January 14, 2014

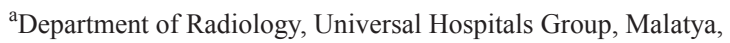
Turkey

${ }^{\mathrm{b}}$ Department of Obstetrics and Gynecology, Kafkas University Medical Faculty, Kars, Turkey

${ }^{\mathrm{c}}$ Department of Radiology, Kafkas University Medical Faculty, Kars, Turkey

${ }^{\mathrm{d}}$ Corresponding author: Murat Bozkurt, Department of Obstetrics and Gynecology, Kafkas University Medical Faculty, Kars, Turkey.

Email: jindrmb@yahoo.com
}

doi: http://dx.doi.org/10.14740/jmc1666w cular pseudo aneurysms. Portal vein diameter greater than 20 $\mathrm{mm}$ is accepted as an aneurysm [2]. Usually, this condition is observed secondary to portal hypertension in patients with chronic hepatic disease. True aneurysms which all wall layers prevented in the absence of chronic hepatic disease and portal hypertension are extremely rare. In this paper, clinical and radiologic findings of incidentally found left liver lobe aplasia and PVA in a 27-week pregnant woman presented with acute abdomen and operated for a right side ovarian torsion are discussed.

\section{Case Report}

A 36-year-old pregnant woman was admitted to our clinic with nausea, bilious vomiting and acute abdomen. Her medical history revealed that her complaints begun 3 days before and she was followed up in another clinic for a preliminary diagnosis of acute appendicitis. In abdominal examination, abdomen was distended and rebound tenderness was present in the right para median region. Obstetric ultrasound revealed a pregnancy of 26 weeks and 6 days, consistent with the last menstrual period. Ultrasound examination was performed with LOGIQ 9 (GE Healthcare Technologies, Ultrasound, Milwaukee, USA) convex (2 - $5 \mathrm{MHz})$ transducer. Obstetric ultrasound revealed a single live intrauterine fetus of 27 weeks with a fetal weight of about $969 \mathrm{~g}$ with cephalic presentation and normal fetal growth. A $78 \times 50 \mathrm{~mm}$ cyst and solid structures compatible with adnex above the cyst were defined on right side ovary. There were no arterial signals detected by a color Doppler sonography (CDS) on solid tissues around the cyst. In abdominal ultrasound, portal vein was larger than normal and its largest diameter was measured as $24 \mathrm{~mm}$ in the distal site adjacent to liver and $21 \mathrm{~mm}$ in the middle site. Hepatic and splenic parenchyma echoes and sizes were in normal ranges. There was no free fluid in abdomen. Splenic vein diameter was measured as $8 \mathrm{~mm}$.

A lower abdominal magnetic resonance imaging (MRI) with a 1.5 T MRI tool (General Electric Signa Excita, GE Health Care System, Milwaukee, USA) revealed a smooth edged cystic lesion on right ovary sized $80 \times 33 \mathrm{~mm}$, which was hypointense on T1-weighted and hyperintense on T2- 


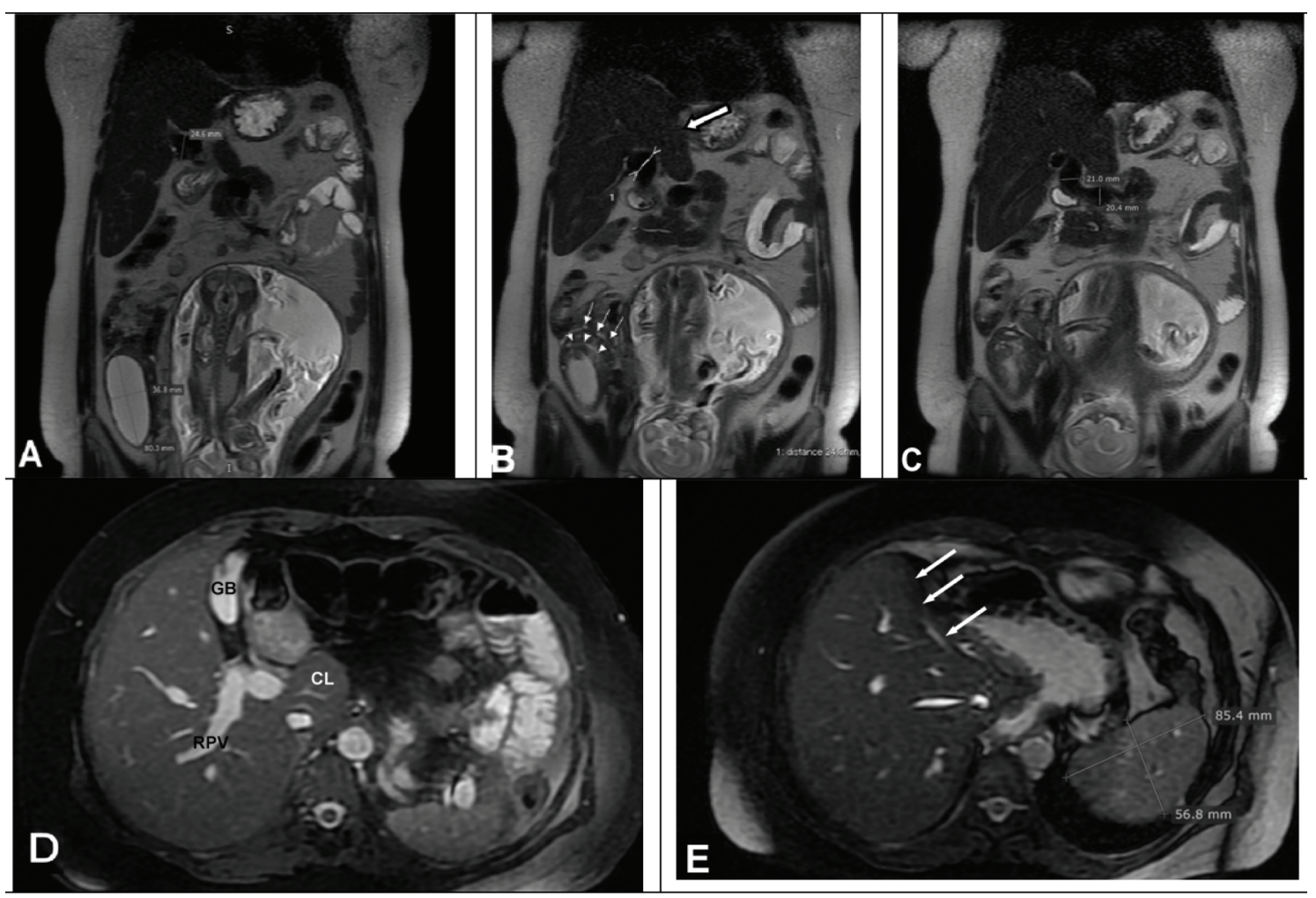

Figure 1. Coronal T2-weighted images (A, B) show a smooth edged hyperintense cystic lesion on right ovary and twisted salpinx (arrow heads and small arrows) was seen on superior aspect of the cyst. The left liver lobe was aplasic (big white arrow). Portal vein was dilated and diameters were measured $24 \mathrm{~mm}$ on distal portion, (C) $21 \mathrm{~mm}$ in the middle part and $20 \mathrm{~mm}$ in proximal portion. (D) Axial fat sat T2-weighted gradient echo image from mid level of liver shows right portal vein (RPV), gall bladder (GB) and caudat lobe (CL). (E) Axial gradient echo fat sat image from upper level of liver shows left liver lob was aplasic (arrows) and spleen sizes were in normal ranges.

weighted series. A solid structure was detected on superior aspect of the cyst which was compatible with at least two times twisted salpinx. Left liver lobe and left portal vein were aplastic and were not visualized. In hepatic hilum, portal vein was divided into two main branches both advancing to the right lobe. Portal vein seemed dilated and its diameter was $24 \mathrm{~mm}$ just before bifurcation, $21 \mathrm{~mm}$ in the middle part and $20 \mathrm{~mm}$ in proximal portion (Fig. 1A-E). Contrast was not used as the patient was pregnant.

These findings suggested an ovarian torsion and patient was taken to operation room with ritodrine treatment for tocolysis. Abdomen was explored through a $4 \mathrm{~cm}$ sized right oblique paramedian incision and a cystic mass of $7 \times$ $7 \mathrm{~cm}$ in size was found. Macroscopic view of the mass was compatible with a hemorrhagic infarct (Fig. 2A, B). With a careful inspection, the mass was observed to be a four-time anti-clockwise twisted ovary and tuba. Salpinx was stuck to ovary and twisted with it. Thus, the tissues were necrotic, and a salphingo-ooferectomy and an appendectomy were performed. Histopathological examination revealed hemorrhagic infarct of the ovarian and tubal tissue (Fig. 2C, D). Postoperative course was uneventful both for the mother and the fetus.
In the postoperative period, patient was examined for chronic liver disease and portal hypertension. There were no esophageal varices on endoscopic examination. There were no laboratory findings consistent with chronic viral hepatitis or chronic liver failure. Patient was discharged on second postoperative day as no findings of portal hypertension have been found as well.

The CDS examination performed after operation, in the 31 st week of gestation revealed no decrease in portal vein diameter. About one month later, the labor, CDS and MRI were repeated. Portal venous diameter was measured as 21 $\mathrm{mm}$ on distal part near the liver, $19 \mathrm{~mm}$ on middle part and $16 \mathrm{~mm}$ on proximal portion (Fig. 3A-C). Partial decrease on portal venous diameter suggested that pregnancy had some effect on portal vein dilation.

\section{Discussion}

Ovarian torsion is a rare cause of acute abdomen and its incidence is $0.05 \%$ [3]. In pregnancy, its incidence is higher than normal population due to ligamentous laxity caused by increased levels of some hormones [4]. Ovarian mass is re- 

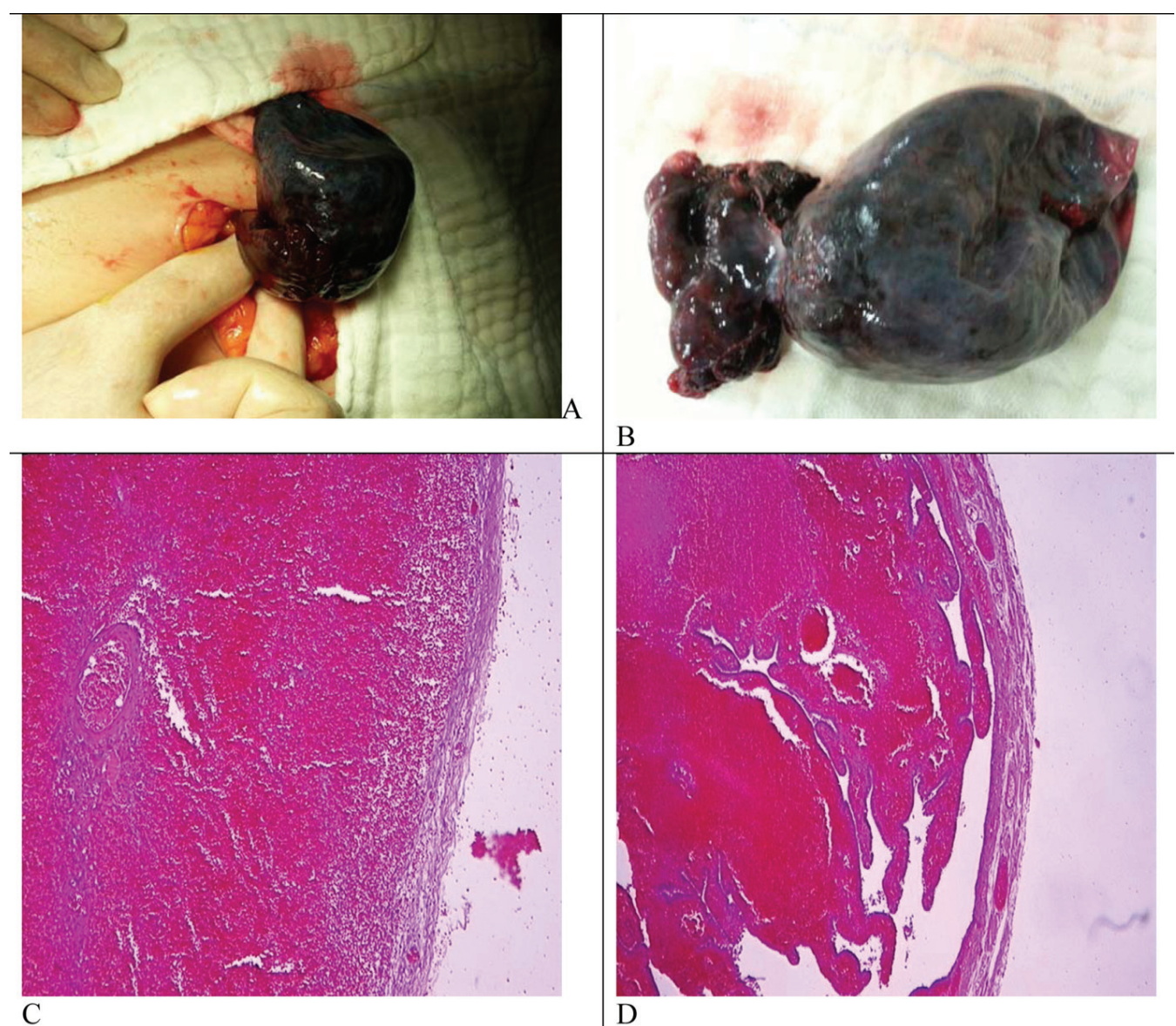

Figure 2. (A) Four-time anti-clockwise twisted ovary and tuba. (B) Cystic mass of $7 \times 7 \mathrm{~cm}$ in size with hemorrhagic infarct. (C) Histopathologic view of ovarian tissues with hemorrhagic and (D) tuba with hemorrhagic infarct.

garded as a risk factor for ovarian torsion. While the most common cause of ovarian torsion in pregnancy is the corpus luteum, all of the ovarian masses may lead to this condition [4]. In our case, pregnancy and cystic mass were considered as risk factors for the ovarian torsion. The pathologic evaluation of the resected cystic lesion was reported as "persistent corpus luteum".

Generally, PVAs occur secondary to portal hypertension. Portal vein diameter may increase up to $19 \mathrm{~mm}$ in cirrhotic patients. Portal vein diameter size greater than $20 \mathrm{~mm}$ at maximum is regarded as PVA [2]. Regardless of whether left liver lobe aplasia causes gastric malpositioning and volvulus, these are generally asymptomatic [1]. To the best of our knowledge, there is no report about PVA associated with left liver lobe aplasia.

In our case, the most important mechanism in the pathogenesis of the PVA development in the absence of chronic hepatic disease is increased retrograde flow due to increased resistance caused by left liver lobe and left portal vein aplasia. Also, decrease in portal vein diameter after labor suggests some gestational effect on this dilation. In a normal gestation, volume of plasma increases by $10 \%$ in the 12 th week and up to $40 \%$ in the middle of the third trimester [5]. Splanchnic blood flow and portal vein blood will naturally increase as well. There are reports showing increased portal vein blood flow in pregnancy [6]. In pregnant women, it is known that some hormones like relaxin (RLX) affect vascular smooth muscles to make dilation [7]. RLX is an insulin-like hormone produced by corpus luteum, placenta and decidua in pregnant women. It converts endothelin (ET) big molecule to ET 1-32 by increasing the gelatinase activity. Produced ET 1-32 provokes endothelial receptor B to produce $\mathrm{NO}$ and make vasodilatation [8]. There are many reports about the effects of RLX on vascular bed and kidneys [9]. However, to the best of our knowledge, there is no report about its effects on portal venous system and liver. Pregnancy does not cause significant portal vein dilation in an individual with a healthy liver. In our case, left liver lobe aplasia caused an increase in the portal vein blood flow and this situation contributed the vein dilation.

As a result, this case shows that patients with left liver lobe aplasia may develop aneurismal portal vein dilation even in the absence of chronic hepatic disease and for these patients portal vein diameter may increase in the course of 


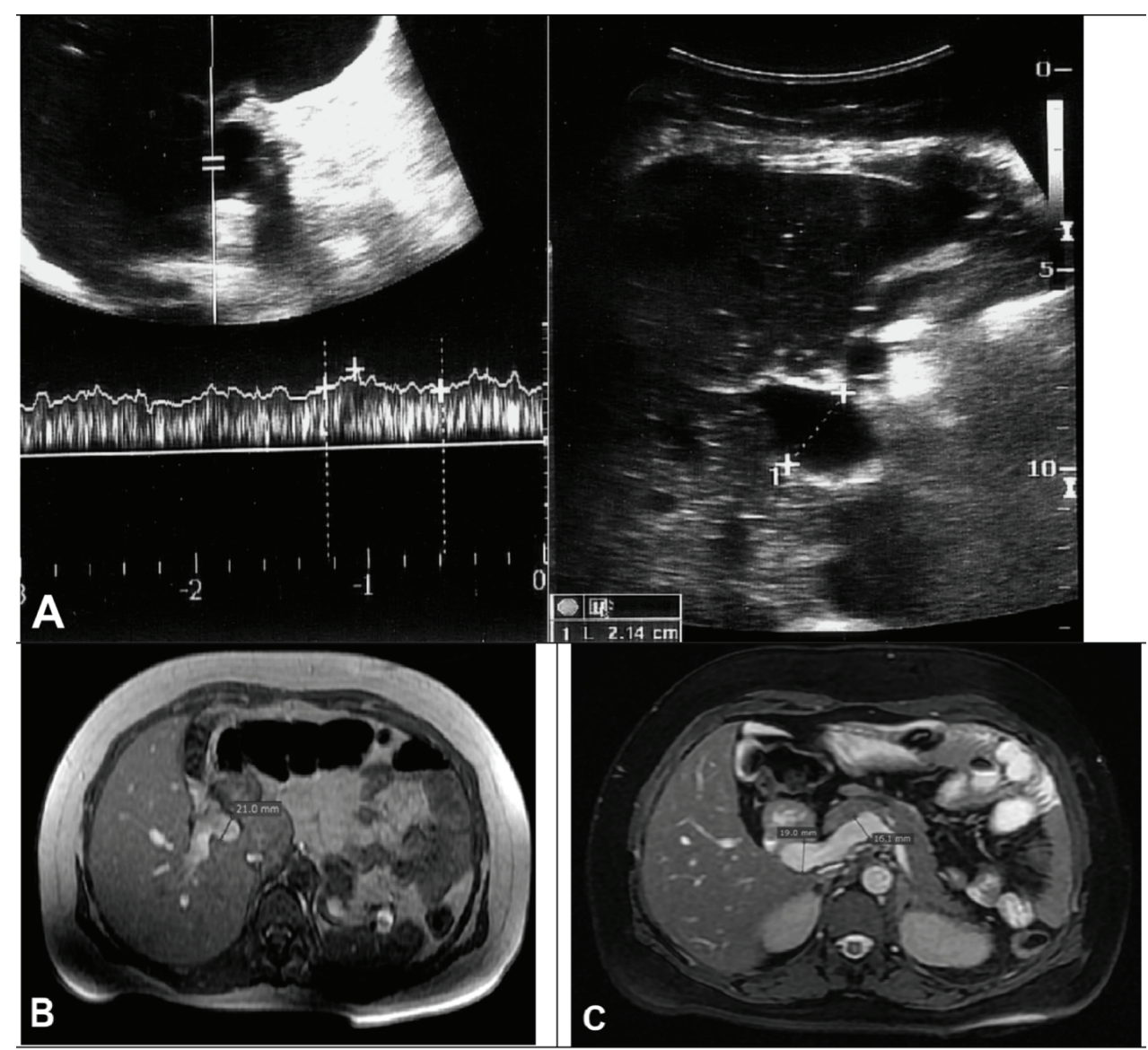

Figure 3. (A) One month after the labor CDS images show hepatopedal blood flow and portal vein diameter was measured $21 \mathrm{~mm}$. (B) Axial T1-weighted gradient echo and (C) gradient echo fat sat MR images show decreased portal vein diameters.

gestation.

\section{References}

1. Aktan Z.A, Savas R, Pinar Y, Arslan O. Lobe And Segment Anomalies of the Liver. J Anat Soc India. 2001;50:15-16.

2. Dognini L, Carreri AL, Moscatelli G. Aneurysm of the portal vein: ultrasound and computed tomography identification. J Clin Ultrasound. 1991;19(3):178-182.

3. Kemmann E, Ghazi DM, Corsan GH. Adnexal torsion in menotropin-induced pregnancies. Obstet Gynecol. 1990;76(3 Pt 1):403-406.

4. Chang HC, Bhatt S, Dogra VS. Pearls and pitfalls in diagnosis of ovarian torsion. Radiographics. 2008;28(5):1355-1368.
5. Chapman AB, Abraham WT, Zamudio S, Coffin C, Merouani A, Young D, Johnson A, et al. Temporal relationships between hormonal and hemodynamic changes in early human pregnancy. Kidney Int. 1998;54(6):20562063.

6. Nakai A, Sekiya I, Oya A, Koshino T, Araki T. Assessment of the hepatic arterial and portal venous blood flows during pregnancy with Doppler ultrasonography. Arch Gynecol Obstet. 2002;266(1):25-29.

7. Conrad KP. Maternal vasodilation in pregnancy: the emerging role of relaxin. Am J Physiol Regul Integr Comp Physiol. 2011;301(2):R267-275.

8. Conrad KP. Mechanisms of renal vasodilation and hyperfiltration during pregnancy. J Soc Gynecol Investig. 2004;11(7):438-448.

9. Davison JM. The kidney in pregnancy: a review. J R Soc Med. 1983;76(6):485-501. 